\title{
A validated liquid chromatography tandem mass spectrometry method for quantification of erlotinib, OSI-420 and didesmethyl erlotinib and semi-quantification of erlotinib metabolites in human plasma
}

Anna Svedberg, Henrik Green, Anders Vikström, Joakim Lundeberg and Svante Vikingsson

\section{Linköping University Post Print}

\section{Tweet}

N.B.: When citing this work, cite the original article.

Original Publication:

Anna Svedberg, Henrik Green, Anders Vikström, Joakim Lundeberg and Svante Vikingsson, A validated liquid chromatography tandem mass spectrometry method for quantification of erlotinib, OSI-420 and didesmethyl erlotinib and semi-quantification of erlotinib metabolites in human plasma, 2015, Journal of Pharmaceutical and Biomedical Analysis, (107), 186-195. http://dx.doi.org/10.1016/j.jpba.2014.12.022

Copyright: Elsevier http://www.elsevier.com/

Postprint available at: Linköping University Electronic Press http://urn.kb.se/resolve?urn=urn:nbn:se:liu:diva-117227 


\section{Title: A validated liquid chromatography tandem mass} spectrometry method for quantification of erlotinib, OSI-420 and didesmethyl erlotinib and semi-quantification of erlotinib metabolites in human plasma

\footnotetext{
Authors: Anna Svedberg ${ }^{a, *}$, Henrik Gréen ${ }^{a, b, c}$, Anders Vikström ${ }^{d}$, Joakim Lundeberg ${ }^{c}$, Svante Vikingsson $^{a}$

${ }^{a}$ Clinical Pharmacology, Division of Drug Research, Department of Medical and Health Sciences, Linköping University, SE-581 85 Linköping, Sweden

${ }^{\mathrm{b}}$ Department of Forensic Genetics and Forensic Toxicology, National Board of Forensic Medicine, SE-587 58 Linköping, Sweden

c School of Biotechnology, Science for Life Laboratory, KTH Royal Institute of Technology, SE-171 21 Solna, Sweden

d Department of Pulmonary Medicine, University Hospital, SE-581 85 Linköping, Sweden

* Corresponding author. Anna Svedberg, Clinical Pharmacology, Division of Drug Research, Department of Medical and Health Sciences, Linköping University, SE-581 85 Linköping, Sweden. Tel.: + 4610 1032184; fax: +4613 149106. E-mail address: anna.svedberg@liu.se
} 


\section{Abstract}

A liquid chromatography tandem mass spectrometry method was developed and validated for quantification of erlotinib and its metabolites in human plasma. The method is suitable for therapeutic drug monitoring and pharmacokinetic studies.

The substances were extracted using protein precipitation, separated on a BEH XBridge $\mathrm{C} 18$ column $(100 \times 2.1 \mathrm{~mm}, 1.7 \mu \mathrm{m})$ by gradient elution at $0.7 \mathrm{~mL} / \mathrm{min}$ of acetonitrile and $5 \mathrm{mM}$ ammonium acetate. The concentration was determined using a Waters Xevo triple quadrupole mass spectrometer in a multi reaction monitoring mode. The total run time was 7 minutes. Deuterated erlotinib and OSI-597 were used as internal standard for erlotinib and its metabolites respectively.

Erlotinib, OSI-420 and didesmethyl erlotinib were quantified in the concentration range 25-5,000 $\mathrm{ng} / \mathrm{mL}, 0.5-500 \mathrm{ng} / \mathrm{mL}$ and $0.15-10 \mathrm{ng} / \mathrm{mL}$ respectively. Precision and accuracy was $<14 \%$ except for OSI-420 at LLOQ (17\%). Extraction recovery was above $89 \%, 99 \%$ and $89 \%$ for erlotinib, OSI-420 and didesmethyl erlotinib respectively.

The human liver microsomes generated 14 metabolites, three of them not previously reported. Twelve metabolites were measured semi-quantitatively and validated with respect to selectivity, precision and stability.

\section{Keywords}

LC-MS/MS; Human liver microsomes; Non-small cell lung cancer; EGFR; Tyrosine kinase inhibitor

\section{Highlights}

- A validated LC-MS/MS method for quantification of erlotinib and its metabolites

- Analysis suitable for TDM and clinical studies

- Identification of fourteen HLM generated metabolites, three not previously reported 


\section{Introduction}

Erlotinib is an epidermal growth factor receptor (EGFR) tyrosine kinase inhibitor (TKI) with the mechanism of action to reversibly bind the ATP pocket of the intra cellular tyrosine kinase and prevent phosphorylation and the following signal cascade usually associated with cellular growth and differentiation [1].

Erlotinib is approved as monotherapy in first line treatment in patients with locally advanced or metastatic non-small cell lung cancer (NSCLC) harboring activating mutations in the EGFR gene. It is also approved in further lines of therapy in NSCLC, regardless of mutational status of the tumor, upon progression after at least one line of chemotherapy. The third indication in NSCLC is as maintenance treatment after at least stable disease following platinum based first line therapy. Erlotinib can also be used in combination with gemcitabine in metastasized pancreatic carcinoma. The recommended dose is $150 \mathrm{mg}$ daily for NSCLC patients [2].

Recently, erlotinib has been suggested as a candidate for therapeutic drug monitoring as extreme inter individual variation has been observed among patients treated with erlotinib [3, 4]. The interindividual variation is mostly caused by genetic factors such as polymorphous transporters and enzymes and other factors such as food intake and smoking [5-9].

Erlotinib is extensively metabolized mainly by the cytochrome P450 (CYP) enzymes CYP3A4, CYP3A5 and CYP1A1 but also by CYP1A2 and CYP2D 6 and only $2 \%$ is eliminated unchanged $[10,11]$. Several metabolites have been identified and the major active metabolite desmethyl erlotinib (OSI-420) has been reported at concentrations around $10 \%$ of erlotinib $[2,12]$. To our knowledge, the importance of the metabolites have not yet been determined.

Today, several validated liquid chromatography methods for quantification of erlotinib and in combination with OSI-420 have been published, using either UV [12-14] or tandem mass spectrometry detection [15-19]. Other techniques such as capillary electrophoresis and fluorescence spectrometry have also been used to develop methods for quantification of erlotinib [20] and three metabolites [21]. However, to our knowledge a method for determining the concentration of other erlotinib metabolites is lacking.

Absolute quantification of erlotinib metabolites is difficult as only a few are commercially available as reference substances. Instead the use of human liver microsomes (HLM), liver homogenate from ER containing all the metabolizing enzymes present in the liver, can be used to generate erlotinib metabolites for semi-quantification of these substances.

The aim of this project was to develop and validate a method for determining the concentration of erlotinib and its metabolites in human plasma, with quantification of erlotinib, OSI-420 and didesmethyl erlotinib and semi-quantification of HLM generated metabolites in human plasma with liquid chromatography tandem mass spectrometry. 


\section{Material and methods}

\subsection{Chemicals and reagents}

Reference standards of erlotinib, desmethyl erlotinib (OSI-420) and didesmethyl erlotinib as well as the internal standards erlotinib-d6 (OSI-774-d3) and 4-methyl erlotinib (OSI-597) were purchased from Toronto Research Chemicals Inc. (North York, ON, Canada).

Ammonium acetate (AmAc) and dimethyl sulfoxide (DMSO), of analytical grade or higher, were obtained from Sigma Aldrich Sweden AB (Solna, Sweden). Acetonitrile (ACN) from Merck AB (Solna, Sweden) and methanol from Fischer Scientific AB (Västra Frölunda, Sweden) were of LC/MS and HPLC grade respectively.

HLM (pool of 200 donors), NADPH regenerating system ( 26 mM NADP ${ }^{+}, 66 \mathrm{mM}$ glucose-6-phosphate, $66 \mathrm{mM} \mathrm{MgCl}_{2}$ and $40 \mathrm{U} / \mathrm{mL}$ glucose-6-phosphate dehydrogenase) and UGT reaction mix ( $25 \mathrm{mM}$ uridin 5'-diphospho-glucuronic acid, $250 \mathrm{mM}$ Tris- $\mathrm{HCl}$ and $40 \mathrm{mM} \mathrm{MgCl}_{2}$ and $0.125 \mathrm{mg} / \mathrm{mL}$ alamethicin) were purchased from BD Bioscience (Stockholm, Sweden).

Drug free EDTA plasma was donated from six volunteers ( 2 males, 4 females) with an average age of $25(22-30)$ years.

\subsection{Chromatographic conditions}

The chromatography system used was an Aquity ultra performance liquid chromatography system with column manager, sample manager and binary solvent manager from Waters, Sollentuna, Sweden. Separation took place on a BEH XBridge C18 column (100 x $2.1 \mathrm{~mm}, 1.7 \mu \mathrm{m}$, Waters) with a $\mathrm{BEH}$ VanGuard C18 pre-column $(5 \times 2.1 \mathrm{~mm}, 1.7 \mu \mathrm{m}$, Waters $)$ at a temperature of $55^{\circ} \mathrm{C}$. The injection volume was $5 \mu \mathrm{L}$. The mobile phase was composed of AmAc $(5 \mathrm{mM})$ and $A C N$ with a flow rate of 0.7 $\mathrm{mL} / \mathrm{min}$. The following gradient elution was used: 10-50 \% ACN (0-5 min), $90 \% \mathrm{ACN}(5-6 \mathrm{~min}), 10 \%$ ACN (6-7 min). Total run time was seven min.

An Aquity Xevo triple quadrupole mass spectrometer (Waters) was used operating in positive electrospray ionization (ESI) mode. The MS parameters were as follows, with a source temperature of $150^{\circ} \mathrm{C}$, desolvation temperature of $600^{\circ} \mathrm{C}$, cone gas flow of $24 \mathrm{~L} / \mathrm{Hr}$, desolvation gas flow of $990 \mathrm{~L} / \mathrm{Hr}$ and argon as collision gas, with a flow of $0.14 \mathrm{~mL} / \mathrm{min}$.

The analytes were monitored in multi reaction monitoring (MRM) mode. Analyte specific parameters are shown in table 1.

\subsection{Sample preparation}

To $50 \mu \mathrm{L}$ plasma, $200 \mu \mathrm{L}$ of cold methanol containing erotinib-d6 and OSI-597 at a concentration of $12.5 \mathrm{ng} / \mathrm{mL}$ was added. After thorough vortex mixing ( $15 \mathrm{~s}$ ) and centrifugation at $10,000 \mathrm{~g}$ for $5 \mathrm{~min}$ at $4^{\circ} \mathrm{C}, 150 \mu \mathrm{L}$ of the supernatant was transferred to a tube containing $150 \mu \mathrm{L} 10 \mathrm{mM}$ AmAc. After vortex mixing ( $5 \mathrm{~min}$ ) the samples were transferred to a $0.7 \mathrm{~mL} 96$-well injection plate.

\subsection{Metabolite generation and identification using human liver microsomes}

HLM generated metabolites were used to optimize parameters for mass spectrometric detection and metabolite quantification. Metabolites were generated by adding cofactors, buffer and analyte (10 $\mu \mathrm{M})$ to a portion of human liver microsomes on ice. The mixture was incubated at $37^{\circ} \mathrm{C}$ for $150 \mathrm{~min}$. The incubation was stopped by the addition of four volumes of methanol and mixed thoroughly before centrifugation at $17,530 \mathrm{~g}$ for $5 \mathrm{~min}$ at $4^{\circ} \mathrm{C}$. The supernatant was transferred to a glass tube and concentrated by evaporation at $37^{\circ} \mathrm{C}$ under nitrogen flow to a volume of approximately $600 \mu \mathrm{L}$. The evaporated concentrate was spiked in drug free plasma to create HLM control samples. Control incubations was performed excluding either erlotinib, cofactors or HLM. 
Table 1: Analyte specific MS parameters

\begin{tabular}{|c|c|c|c|c|c|c|}
\hline Analytes & $\begin{array}{l}\text { Retention } \\
\text { time } \\
\text { (min) }\end{array}$ & $\begin{array}{c}\text { Precursor } \\
\text { ion } \\
(\mathrm{m} / \mathrm{z})\end{array}$ & $\begin{array}{c}\text { Cone } \\
\text { voltage } \\
\text { (V) }\end{array}$ & $\begin{array}{l}\text { Product } \\
\text { ion }^{\mathrm{a}} \\
(\mathrm{m} / \mathrm{z})\end{array}$ & $\begin{array}{c}\text { Collision } \\
\text { energy } \\
(\mathrm{eV})\end{array}$ & $\begin{array}{l}\text { Dwell } \\
\text { time } \\
\text { (ms) }\end{array}$ \\
\hline \multirow[t]{2}{*}{ M2 } & 1.36 & 414.2 & 48 & 356.2 & 25 & 75 \\
\hline & & & & 312.2 & 30 & 75 \\
\hline \multirow[t]{2}{*}{ M3 } & 1.81 & 586.2 & 43 & 410.1 & 30 & 50 \\
\hline & & & & 352.2 & 40 & 50 \\
\hline \multirow[t]{2}{*}{ M6 } & 1.87 & 428.2 & 48 & 312.2 & 30 & 50 \\
\hline & & & & 370.2 & 25 & 50 \\
\hline \multirow[t]{2}{*}{$\mathrm{M} 20^{\mathrm{b}}$} & 2.18 & 586.3 & 43 & 410.1 & 30 & 50 \\
\hline & & & & 351.9 & 40 & 50 \\
\hline \multirow[t]{2}{*}{ M17 } & 2.31 & 396.2 & 38 & 294.2 & 30 & 40 \\
\hline & & & & 338.2 & 25 & 40 \\
\hline \multirow[t]{2}{*}{$\mathrm{M} 8 / 10$} & 2.33 & 556.2 & 43 & 321.9 & 45 & 40 \\
\hline & & & & 380.4 & 25 & 40 \\
\hline \multirow[t]{2}{*}{$\mathrm{M} 21^{\mathrm{b}}$} & 2.34 & 396.2 & 38 & 294.2 & 30 & 40 \\
\hline & & & & 338.2 & 25 & 40 \\
\hline \multirow[t]{2}{*}{ M7 } & 2.41 & 430.2 & 48 & 296.2 & 35 & 40 \\
\hline & & & & 372.2 & 25 & 40 \\
\hline \multirow[t]{2}{*}{ M11 } & 2.62 & 394.2 & 43 & 276.2 & 35 & 50 \\
\hline & & & & 290.2 & 35 & 50 \\
\hline \multirow[t]{2}{*}{ M19b } & 2.87 & 428.2 & 42 & 312.2 & 30 & 30 \\
\hline & & & & 370.2 & 30 & 30 \\
\hline \multirow{2}{*}{$\begin{array}{c}\text { Didesmethyl } \\
\text { erlotinib }\end{array}$} & 3.06 & 366.2 & 42 & 278.2 & 30 & 75 \\
\hline & & & & 101.2 & 50 & 75 \\
\hline \multirow[t]{2}{*}{ M16 } & 3.07 & 410.2 & 43 & 294.2 & 30 & 50 \\
\hline & & & & 352.2 & 25 & 25 \\
\hline \multirow[t]{2}{*}{ OSI-420 } & 3.56 & 380.2 & 42 & 278.2 & 30 & 75 \\
\hline & & & & 322.2 & 24 & 75 \\
\hline \multirow[t]{2}{*}{ M13 } & 3.67 & 380.2 & 42 & 278.2 & 30 & 75 \\
\hline & & & & 336.2 & 24 & 75 \\
\hline \multirow[t]{2}{*}{ Erlotinib } & 4.35 & $395.2 c$ & 13 & 279.2 & 43 & 25 \\
\hline & & & & 337.2 & 28 & 25 \\
\hline \multirow[t]{2}{*}{ Erlotinib-d6 } & 4.32 & 400.2 & 44 & 278.2 & 30 & 75 \\
\hline & & & & 339.2 & 22 & 75 \\
\hline \multirow[t]{2}{*}{ OSI-597 } & 4.78 & 408.1 & 50 & 292.1 & 30 & 128 \\
\hline & & & & 350.2 & 24 & 128 \\
\hline \multicolumn{7}{|c|}{ Qualification ions are listed below the quantification ions } \\
\hline \multicolumn{7}{|l|}{ Novel metabolite } \\
\hline C13-isotope ion & & & & & & \\
\hline
\end{tabular}


HLM generated metabolites were either identified with a targeted approach based on existing literature [11] or with an untargeted approach were potential metabolites were searched for in a full scan in the $200-600 \mathrm{~m} / \mathrm{z}$ range. Potential metabolite masses with a peak significant above noise in the full scan were further studied in a daughter scan. The two most abundant fragments in the daughter scan were chosen as quantification and qualification fragments. Cone voltage and collision energy were optimized using stepwise modifications for each metabolite.

\subsection{Calibration and quality control}

Stock solutions of erlotinib, OSI-420, didesmethyl erlotinib, erlotinib-d6 and OSI-597 were prepared in DMSO, working solutions were further prepared by dilution with $\mathrm{MeOH}: \mathrm{H} 2 \mathrm{O}(50: 50 ; \mathrm{v} / \mathrm{v})$ and stored at $-20^{\circ} \mathrm{C}$.

Calibration standards were prepared at six levels in drug free plasma for erlotinib, OSI-420 and didesmethyl erlotinib in the concentration range of $25-5,000 \mathrm{ng} / \mathrm{mL}$ (25-75-300-1,000-3,750-5,000), $0.5-500 \mathrm{ng} / \mathrm{mL}$ (0.5-1.5-10-75-375-500) and 0.15-10 ng/mL (0.15-0.45-1-3-7.5-10), respectively. Quality control (QC) samples were prepared in five concentration levels (LLOQ-low-medium-medhighhigh) in drug free plasma for erlotinib (25-75-400-1,200-3,750 ng/mL), OSI-420 (0.5-1.5-20-100-375 $\mathrm{ng} / \mathrm{mL}$ ) and didesmethyl erlotinib (0.15-0.45-1.5-4-7.5 mg/mL). A calibration model of linear regression (weighting $1 / \mathrm{x}$ ) was used for OSI-420 and didesmethyl erlotinib. For erlotinib a calibration model with quadratic regression (In transformation) was used.

Lacking commercial reference substances, M2, M6, M7, M8/10, M11, M17, M19, M20 and M21 were quantified using the calibration curve of didesmethyl erlotinib and M3, M13 and M16 were quantified using the calibration curve of OSI-420.

\subsection{Method validation}

The method was validated according to guidelines from the Food and Drug Administration [22] and the European Medicines Agency [23] for erlotinib, OSI-420 and didesmethyl erlotinib. Due to the lack of reference standard, a limited validation (selectivity, precision and stability) was performed for the HLM generated metabolites, M2, M3, M6, M7, M8/10, M11, M13, M16, M17, M19, M20 and M21.

Selectivity was determined in drug free plasma from six different individuals. The area of interfering peaks should be $<20 \%$ of LLOQ or $<5 \%$ for the internal standards to be considered acceptable. Carryover was determined by analyzing a blank sample after the highest calibration standard. The blank sample should contain $<20 \%$ of LLOQ or $<5 \%$ for the internal standards.

The suitability of the calibration models were evaluated by back calculating the calibration curves. All points should be within $\pm 15 \%$ of the nominal value except for LLOQ that should be within $\pm 20 \%$ of the nominal value.

Intra- $(n=5)$ and inter-day $(n=11)$ accuracy and precision for erlotinib, OSI-420 and didesmethyl erlotinib was determined at five QC levels. Accuracy and precision should be within $\pm 15 \%$ except for LLOQ that should be within $\pm 20 \%$. Intra-day precision $(n=5)$ was determined for HLM produced metabolites at low and high concentration levels. Inter-day precision was determined in six samples analysed on three different days.

Dilution integrity of erlotinib, OSI-420 and didesmethyl erlotinib was determined using drug free plasma spiked at $10 \times$ ULOQ $(50,000 \mathrm{ng} / \mathrm{mL}$ erlotinib, 5,000 ng/mL OSI-420 and $100 \mathrm{ng} / \mathrm{mL}$ didesmethyl erlotinib) that was diluted $40 \mathrm{x}$ with drug free plasma $(n=5)$. Accuracy and precision should be within $\pm 15 \%$.

Matrix effects were studied by analyzing drug free plasma from six individuals spiked at low and high QC levels. The matrix effect was determined as the ratio between internal standard corrected area in 
a sample spiked after extraction and a solution of $40 \%$ methanol in $10 \mathrm{mM}$ AmAc. Matrix effects were considered acceptable if CV $<15 \%$ [23].

Extraction recovery was evaluated at low, medium and high QC levels $(n=3)$ by internal standard corrected area in samples spiked before and after extraction.

Stability was evaluated for erlotinib, OSI-420 and didesmethyl erlotinib at low, medium and high QC levels $(n=3)$. Short term stability was determined after storage of spiked plasma samples at room temperature for $24 \mathrm{~h}$. Freeze-thaw stability was determined after three freeze-thaw cycles. Freezethaw stability was also determined for HLM generated metabolites at two concentration levels $(n=3)$. Processed sample stability was determined by reinjection of a calibration curve and QC samples stored at $+4^{\circ} \mathrm{C}$ after 8,24 and $72 \mathrm{~h}$. Long term stability was determined after storage for 12 weeks at $-20^{\circ} \mathrm{C}$. The analytes were considered stable if accuracy was within $\pm 15 \%$ of the nominal value. Long term stability for HLM generated metabolites was determined after 10 weeks in patient samples $(n=2)$ and considered stable if $\mathrm{CV}<15 \%$ in each patient $(n=3)$.

\subsection{Patient samples}

Blood samples were collected from patients with non-small cell lung cancer after one and two months on erlotinib regimen. Erlotinib, $150 \mathrm{mg}$, was administered once daily and blood samples were collected in $7 \mathrm{~mL}$ EDTA tubes $22-26 \mathrm{~h}$ after last dose. Samples were centrifuged at $1350 \mathrm{~g}$ for $10 \mathrm{~min}$ at $4^{\circ} \mathrm{C}$ and plasma was transferred to a tube and stored at $-70^{\circ} \mathrm{C}$ until analysis.

The study protocol was approved by the ethical review board in Linköping, Sweden (DNR 2013/20131 ) and the patients provided a written informed consent prior to study entry.

Table 2: Intra- and inter-batch precision and accuracy

\begin{tabular}{|c|c|c|c|c|c|c|}
\hline \multirow[b]{3}{*}{ Analytes } & & & \multicolumn{2}{|c|}{ Intra-batch $(n=5)$} & \multicolumn{2}{|c|}{ Inter-batch $(n=11)$} \\
\hline & \multirow{2}{*}{\multicolumn{2}{|c|}{$\begin{array}{r}\text { Nominal conc. } \\
(\mathrm{ng} / \mathrm{mL})\end{array}$}} & Precision & Accuracy & Precision & Accuracy \\
\hline & & & (\%) & $(\%)$ & (\%) & $(\%)$ \\
\hline \multirow[t]{5}{*}{ Erlotinib } & LLOQ & 25 & 7.6 & 94 & 9.0 & 102 \\
\hline & Low & 75 & 5.9 & 95 & 5.2 & 95 \\
\hline & Medium & 400 & 2.6 & 95 & 4.1 & 96 \\
\hline & Med-High & 1200 & 1.1 & 97 & 5.6 & 97 \\
\hline & High & 3750 & 4.0 & 101 & 4.4 & 97 \\
\hline \multirow[t]{5}{*}{ OSI-420 } & LLOQ & 0.50 & 9.3 & 108 & 17 & 99 \\
\hline & Low & 1.5 & 3.1 & 102 & 4.2 & 104 \\
\hline & Medium & 20 & 1.6 & 97 & 4.2 & 102 \\
\hline & Med-High & 100 & 1.3 & 104 & 3.3 & 107 \\
\hline & High & 375 & 1.4 & 101 & 5.5 & 99 \\
\hline \multirow{5}{*}{$\begin{array}{c}\text { Didesmethyl } \\
\text { erlotinib }\end{array}$} & LLOQ & 0.15 & 8.0 & 96 & 11 & 103 \\
\hline & Low & 0.45 & 9.6 & 104 & 14 & 105 \\
\hline & Medium & 1.5 & 5.1 & 100 & 3.8 & 105 \\
\hline & Med-High & 4.0 & 2.9 & 104 & 5.5 & 106 \\
\hline & High & 7.5 & 3.3 & 107 & 8.1 & 104 \\
\hline
\end{tabular}




\section{Results}

A method for quantification of erlotinib and 14 metabolites was developed and validated. Typical chromatograms are shown in figures 1 and 2.

\subsection{Metabolite identification}

The human liver microsomes generated 14 different metabolites, eleven oxidative metabolites (M2, M6, M7, M11, M12, M13, M14, M16, M17, M19 and M21) and three glucuronides (M3, M8/10 and $M 20$ ), three of which are not previously reported (M19, M20 and M21), figure 2. Optimized MRM settings can be found in table 1 . The metabolite structures and biotransformation pathways were illustrated in figure 5 .

Elucidation of new metabolite structures was made from chromatograms and product ion mass spectra. Erlotinib and its metabolites share fragmentation loss at position R1 and or R2, figure 3. M19 and M20 share the same precursor and product ions as M6 and M3 respectively supporting a structural correlation, figure 4 . The almost identical fragmentation pattern makes it difficult to accurately distinguish the structures from each other.

We suggest that the structures of M6 and M19 are hydroxylated erlotinib metabolites with the phenylacetylene group transformed to an acetophenone group. Different positions of the hydroxyl group in the main structure could explain the existence of more than one structure, figure 4.
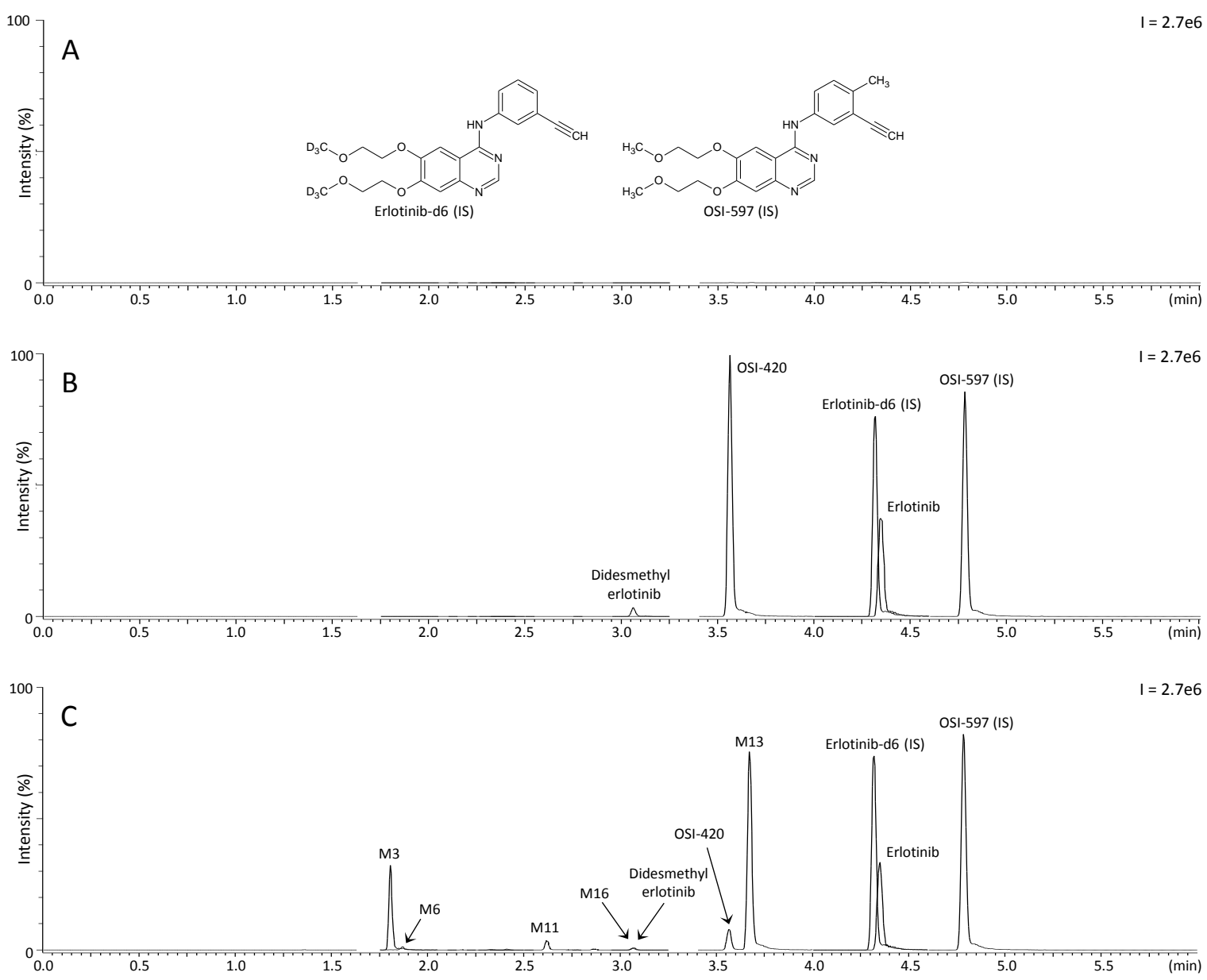

Figure 1: Chromatograms representing a blank plasma sample (A), a medhigh QC sample (B) and a patient sample after 1 month of erlotinib treatment (C). Structures of the internal standards erlotinib-d6 and OSI-597 are presented in A. 
M20 and M3 are probably glucuronides differing only in the position of the glucuronide, figure 4 . The positional isomers M20 and M3 suggests the presence of positional isomers for M16, as a precursor to the glucuronidation.

The structure and identity of M17 and M21 is suggested from correlation in mass spectra and relative retention times from the structurally known isomers OSI-420 (3.56 min) and M13 (3.67 min), figure 3. OSI-420 have a major loss of methoxyethane at R2 $(\mathrm{m} / \mathrm{z} 322)$ and minor loss of ethanol at R1 $(\mathrm{m} / \mathrm{z}$ 336), same pattern as for the $2.31 \mathrm{~min}$ analyte $(\mathrm{m} / \mathrm{z} 338$ and $\mathrm{m} / \mathrm{z} 352)$ while M13 show a more even distribution between methoxyethane and ethanol losses at R1 and R2 respectively, same as the 2.34 min analyte. We therefore suggest that the previously proposed structure of M17 [11], alike OSI-420, is the 2.31 min eluting analyte and M21 is the 2.34 min eluting analyte. The position isomers of M8 and M10 could not be separated and are reported as one, M8/10.

\subsection{Method validation}

The method was successfully validated according to international guidelines.

\subsubsection{Selectivity and carry-over}

No interfering peaks were identified in drug free plasma from different individuals $(n=6)$. No carryover was observed for erlotinib or didesmethyl erlotinib. However, a carry-over of $0,073 \%$ was observed for OSI-420 (corresponding to $73 \%$ of LLOQ).

\subsubsection{Calibration curves}

The simplest calibration model for OSI-420 and didesmethyl erlotinib was a linear regression (weighing $1 / x$ ) but for erlotinib a quadratic regression (In axis transformation) had to be used due to signal saturation and wide range.

Table 3: HLM control intra- and inter-batch precision

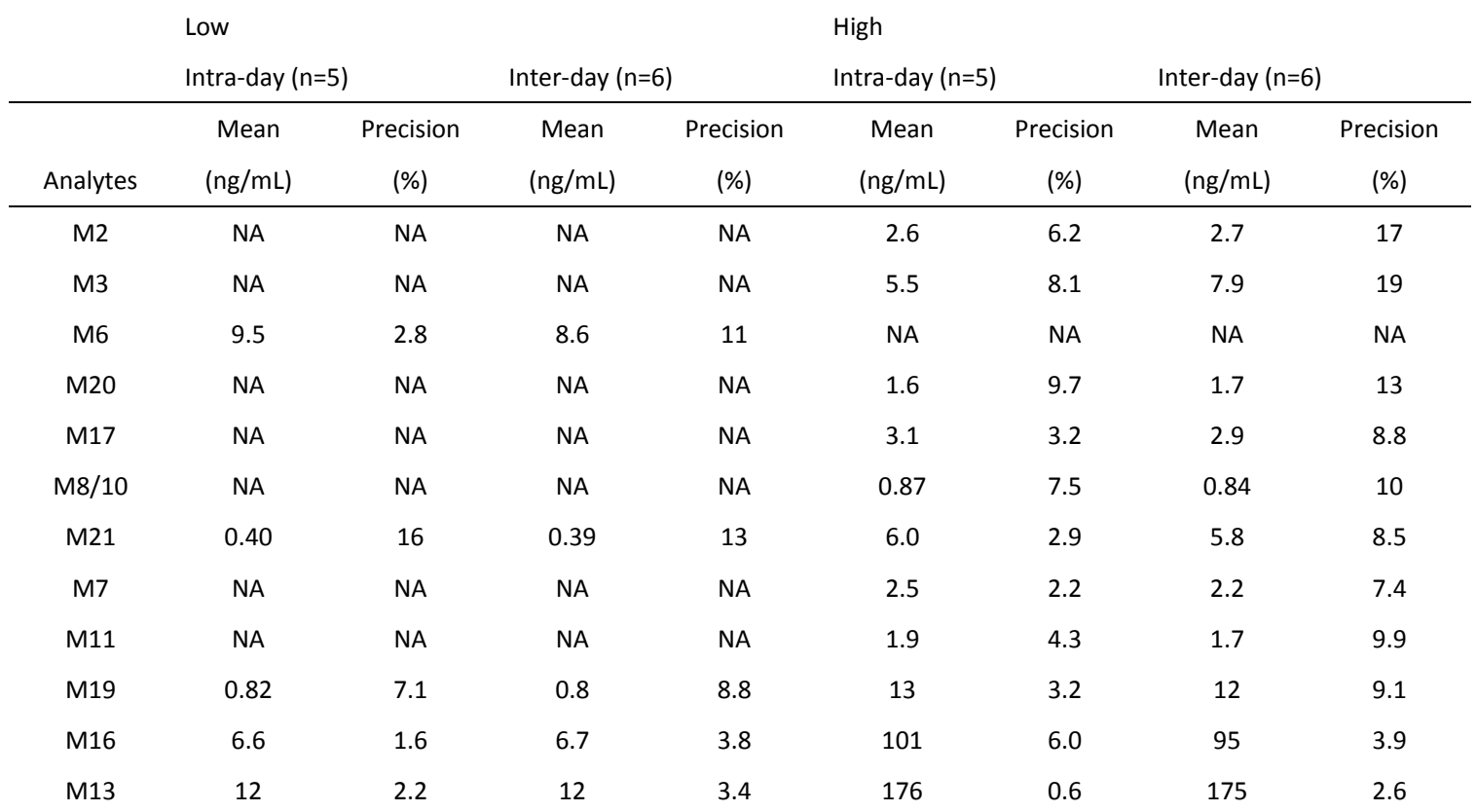

NA: Above or below ULOQ or LLOQ respectively 

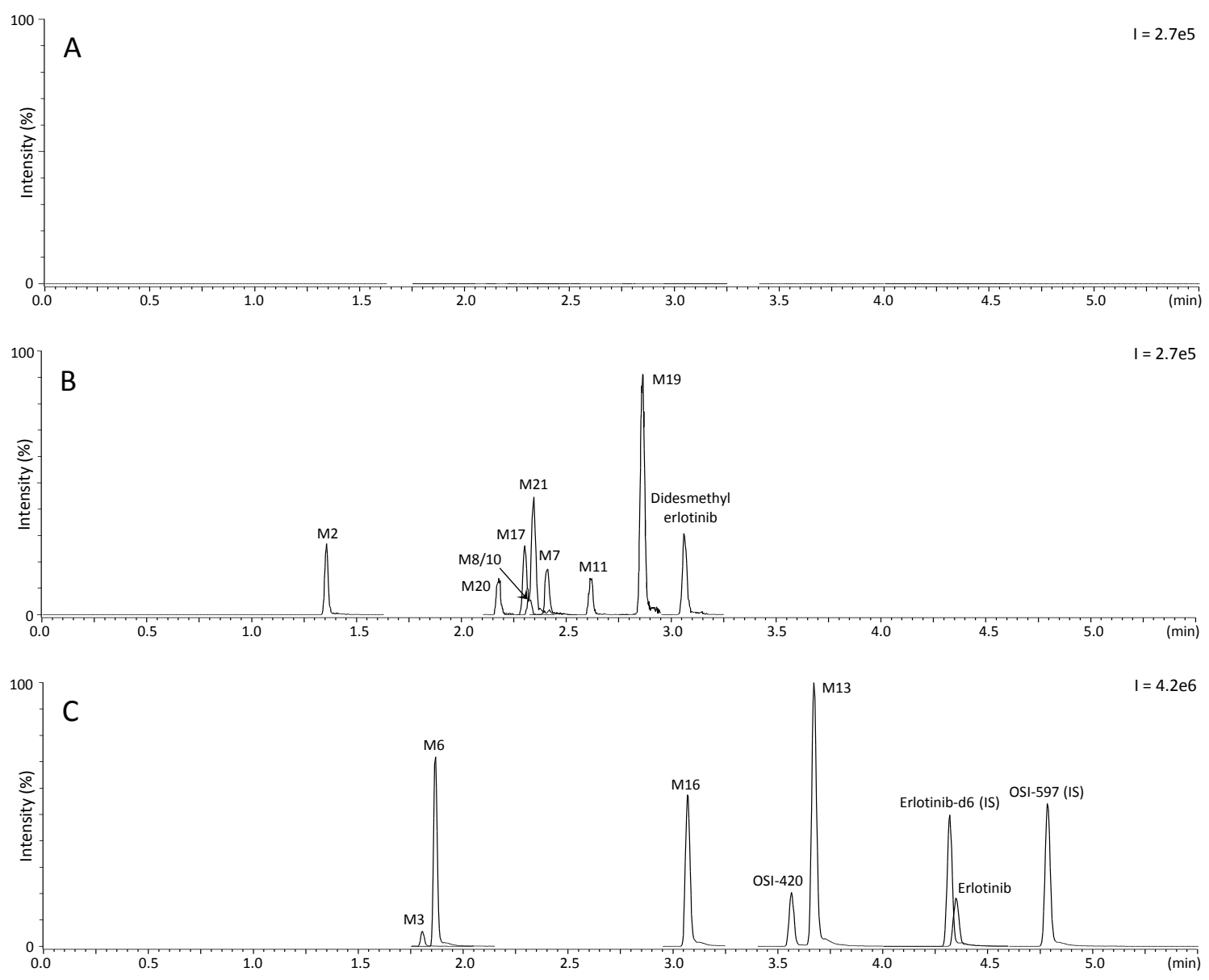

Figure 2: HLM generated metabolites after 150 min incubation. HLM control without erlotinib (A), minor metabolites (B) and major metabolites including the internal standards, erlotinib-d6 and OSI-597 (C).

\subsubsection{Precision, accuracy and dilution integrity}

Intra-batch precision and accuracy $(n=5)$ for erlotinib, OSI-420 and didesmethyl erlotinib was $<10 \%$ and within $\pm 8 \%$ respectively for all concentration levels, table 2 .

Inter-batch precision ( $n=12$ ) was less than $9 \%, 6 \%$ and $14 \%$ for erlotinib, OSI-420 and didesmethyl erlotinib, respectively, except for OSI-420 at LLOQ (17\%). Inter-batch accuracy $(n=12)$ for erlotinib, OSI-420 and didesmethyl erlotinib was within $\pm 7 \%$.

Intra-batch precision for HLM generated metabolites was within $10 \%$ except for M21 with a precision of $16 \%$, table 3 . Inter-batch precision was $<15 \%$ for HLM generated metabolites except for the high concentration of $\mathrm{M} 2$ and $\mathrm{M} 3$ with an inter-batch precision $<20 \%$.

Precision and accuracy for dilution integrity $(n=5)$ was $<3 \%$ and within $\pm 11 \%$ respectively for erlotinib, OSI-420 and didesmethyl erlotinib supporting a dilution of samples with concentrations above ULOQ.

\subsubsection{Matrix effects and recovery}

Matrix effects, at low QC was $99.3 \pm 3.8 \%, 69.7 \pm 5.6 \%, 80.3 \pm 4.6 \%$ for erlotinib, OSI-420 and didesmethyl erlotinib respectively $(n=6)$. Matrix effects at high QC were $96.7 \pm 0.9 \%, 88.3 \pm 0.7 \%$ and $91.1 \pm 2.1 \%$ for erlotinib, OSI-420 and didesmethyl erlotinib respectively. Matrix effects were observed in OSI-420 and didesmethyl erlotinib but the effects were consistent between individuals 
(CV < 6\%). Extraction recovery for erlotinib, OSI-420 and didesmethyl erlotinib was above $89 \%, 99 \%$ and $89 \%$ respectively.
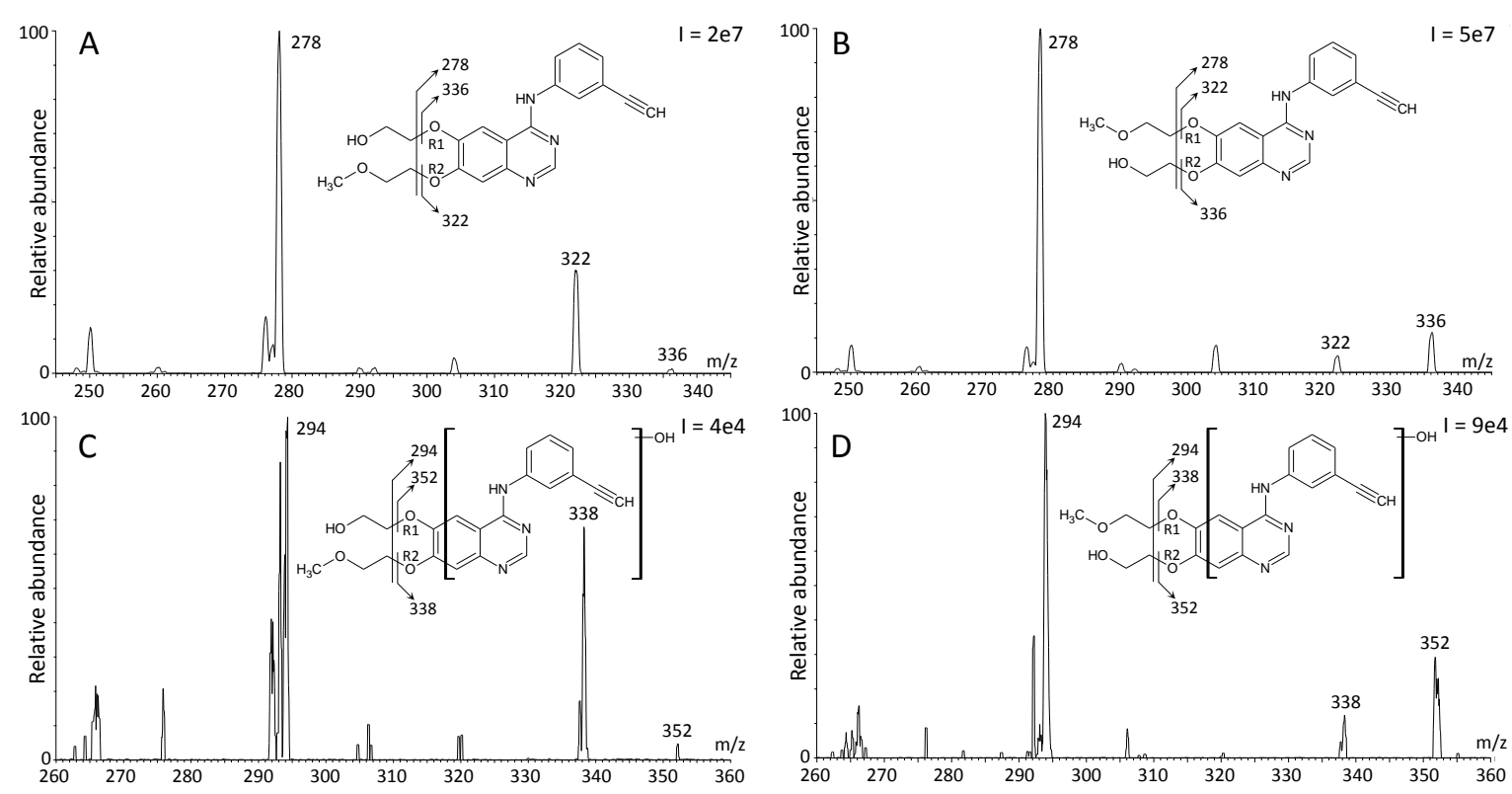

Figure 3: Structure elucidation of M17 and the newly identified M21 by comparing product ion scan mass spectra and retention time with the structurally known OSI-420 and M13. OSI-420 (A) and M17 (C) have a great loss of methoxyethane at $R 2$ ( $\mathrm{m} / \mathrm{z} 322$ and $\mathrm{m} / \mathrm{z} 338$ respectively), a minor loss of ethanol at $R 1(\mathrm{~m} / \mathrm{z} 336$ and $\mathrm{m} / \mathrm{z} 352$ respectively) and elutes prior to their corresponding isomers supporting an identical arrangement of the methoxyethane and methanol in the OSI-420 and M17 metabolic structures. M13 (B) and M21 (D) show a balanced distribution between $R 1(322 \mathrm{~m} / z$ and $338 \mathrm{~m} / \mathrm{z})$ and $R 2(336 \mathrm{~m} / z$ and $352 \mathrm{~m} / \mathrm{z})$ losses indicating that ethanol and methoxyethane are arranged identically in the M13 and M21 metabolic structures.
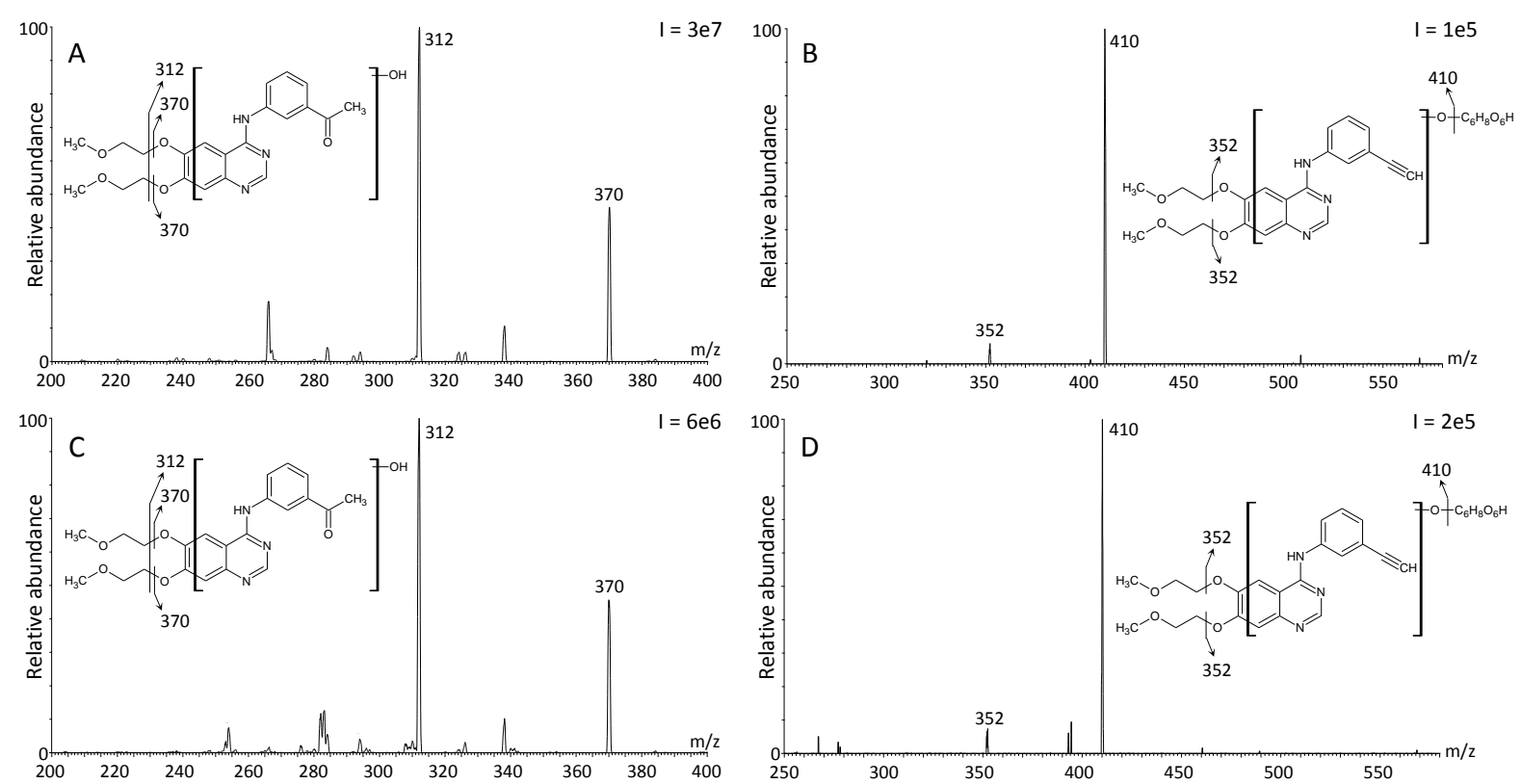

Figure 4: Proposed structures of the newly identified metabolites M19 and M20 by comparing product ion scan mass spectra with M6 and M3. M6 (A) and M19 (C) as well as M3 (B) and M20 (D) show similar fragmentation pattern indicating that the newly identified metabolites M19 and M20 are isomers to M6 and M3 respectively. 

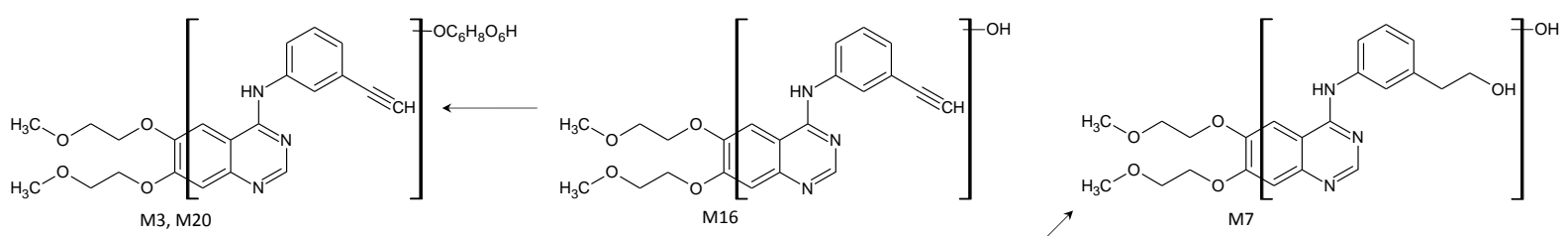<smiles>COCCOc1cc2ncnc(Nc3cccc(C#CCO)c3)c2cc1OCCO</smiles><smiles>C#Cc1cccc(Nc2ncnc3cc(OCCOC)c(OCCOC)cc23)c1</smiles>

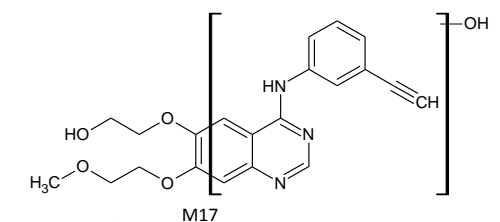

** Demethylation and glucuronidation

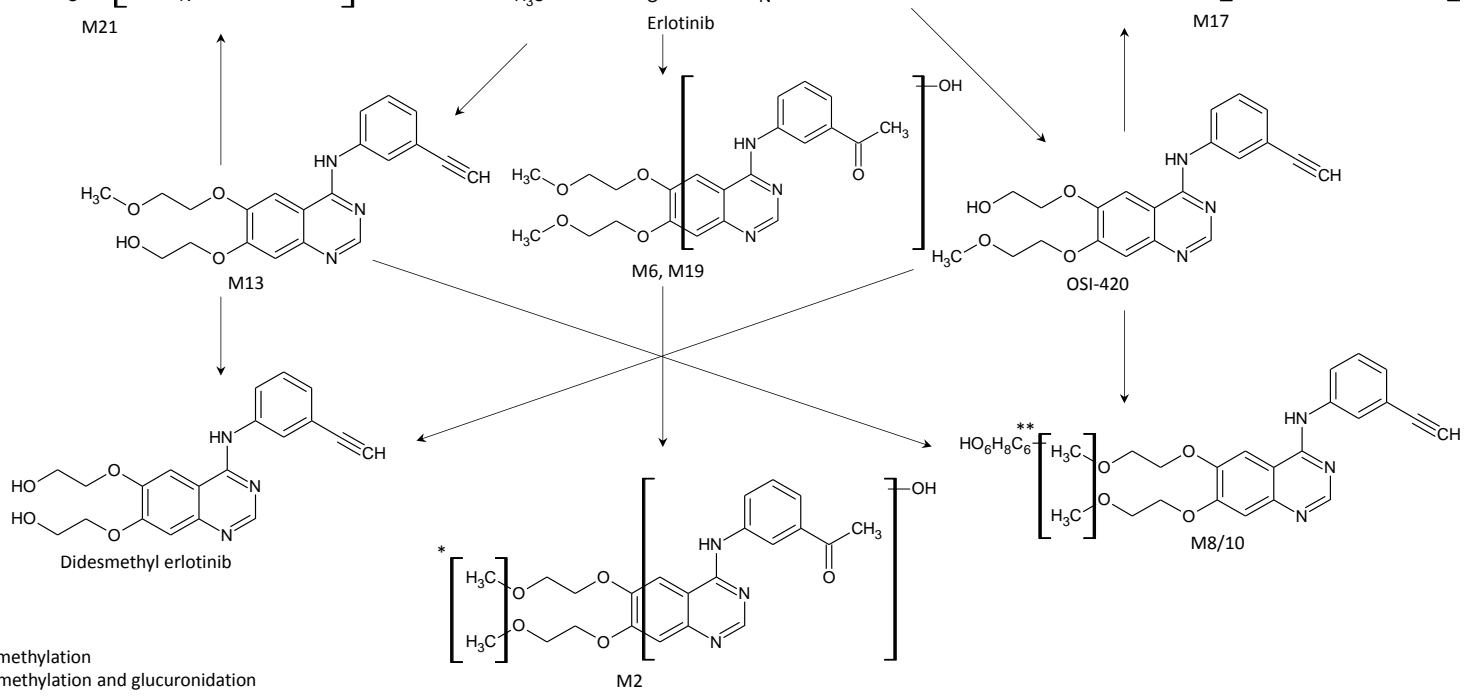

Figure 5: Proposed metabolite structures and biotransformation pathways of erlotinib.

\subsubsection{Stability}

Erlotinib, OSI-420 and didesmethyl erlotinib were stable in plasma for $24 \mathrm{~h}$ at room temperature, for three freeze-thaw cycles and for at least 3 months at $-20^{\circ} \mathrm{C}$ storage, table 4 . Processed samples were stable for $24 \mathrm{~h}$ at $5^{\circ} \mathrm{C}$. HLM generated metabolites were stable for three freeze-thaw cycles $(n=3)$ at low and high control levels. Erlotinib metabolites above LLOQ (M3, M11, M16, M6, M19, M7, M13) in patient plasma samples $(n=2)$ were stable for 10 weeks at $-80^{\circ} \mathrm{C}(\mathrm{CV}<15 \%, \mathrm{n}=3)$, except from M3. Increased variability was observed for M11 (CV $27 \%$ and $30 \%$ ), M16 (CV $16 \%$ and $18 \%$ ) and M7 (CV $20 \%$ and $10 \%)$. However, no increasing or decreasing trends were observed for M11, M16 or M7.

Table 4: Stability of erlotinib, OSI-420 and didesmethyl erlotinib $(n=3)$

\begin{tabular}{ccccc}
\hline Analytes & $\begin{array}{c}\text { Nominal } \\
\text { conc. (ng/mL) }\end{array}$ & $\begin{array}{c}\mathrm{t}=24 \mathrm{~h} \mathrm{RT} \\
\text { pre-prep. (\%) }\end{array}$ & $\begin{array}{c}\text { 3 freeze-thaw } \\
\text { cycles (\%) }\end{array}$ & $\begin{array}{c}\mathrm{t}=3 \text { months } \\
-20^{\circ} \mathrm{C}(\%)\end{array}$ \\
\hline Erlotinib & 75 & 101 & 95 & 95 \\
& 400 & 97 & 95 & 99 \\
& 3750 & 92 & 96 & 96 \\
OSI-420 & 1.5 & 96 & 109 & 109 \\
& 20 & 98 & 104 & 106 \\
& 375 & 90 & 93 & 92 \\
Didesmethyl & 0.45 & 104 & 95 & 96 \\
erlotinib & 1.5 & 101 & 104 & 112 \\
& 7.5 & 92 & 97 & 104
\end{tabular}




\subsubsection{Clinical application}

All metabolites, except for M17, were detected in patient samples $(n=4)$ at varying concentrations, table 5. M20 was detected but below LLOQ. A typical chromatogram is shown in figure 1C.

Table 5: Patient concentrations ( $n=4)$

\begin{tabular}{cccc}
\hline Major & $\begin{array}{c}\text { Mean (range) } \\
(\mathrm{ng} / \mathrm{mL})\end{array}$ & Minor & $\begin{array}{c}\text { Mean }(\mathrm{range}) \\
(\mathrm{ng} / \mathrm{mL})\end{array}$ \\
\hline Erlotinib & $1100(690-1800)$ & $\mathrm{M} 6$ & $2.3(1.5-3.7)$ \\
M13 & $110(72-180)$ & $\mathrm{M} 19$ & $0.71(0.49-0.93)$ \\
M3 & $71(37-140)$ & $\mathrm{M} 7$ & $0.54(0.36-0.73)$ \\
OSI-420 & $18(8.4-26)$ & $\mathrm{M} / 10$ & $0.30(0.17-0.62)$ \\
M11 & $5.1(4.6-6.2)$ & $\mathrm{M} 21$ & $0.18(-0.28)$ \\
M16 & $2.9(1.2-4.5)$ & $\mathrm{M} 2$ & $0.15(-0.24)$ \\
Didesmethyl & $2.3(0.86-4.2)$ & $\mathrm{M} 20$ & Below LLOQ \\
erlotinib & & $\mathrm{M} 17$ & Not detected
\end{tabular}




\section{Discussion}

We present a sensitive and simple method for quantification of erlotinib, OSI-420 and didesmethyl erlotinib and for semi-quantification of HLM generated metabolites.

The simultaneous monitoring of all analytes was enabled by sub optimization of erlotinib collision energy and detection of the isotope ion (m/z 395.2 instead of $\mathrm{m} / \mathrm{z} 394.2)$. Signal saturation was observed for erlotinib and erlotinib- $\mathrm{d} 6$ at high concentrations. To avoid a saturated internal standard affecting the metabolite quantification, OSI-594 was used as internal standard for all metabolites.

Due to the saturation of erlotinib, the most suitable calibration model was a quadratic regression with In axis transformation. Linear regression $(1 / x)$ was used for OSI-420 and didesmethyl erlotinib.

The HLM generated metabolites were quantified using the calibration of either OSI-420 or didesmethyl erlotinib depending on the expected levels in patient samples. Even though accurate metabolite concentrations cannot be assured as the accuracy of the method cannot be determined without reference standards the high precision allows for comparison between patients.

OSI-420, considered to be the major erlotinib metabolite, is reported separately from its position isomer M13 in this method, figure 2C. But the results from HLM and patient samples indicates that the major erlotinib metabolite is $\mathrm{M} 13$, figure $1 \mathrm{C}$ and $2 \mathrm{C}$. M13 have a 6-fold higher average concentration than OSI-420, consistent with the previously reported peak height ratio in rat liver [24]. The certificate of analysis of the reference substance only contains MS and H-NMR data which cannot be readily used to distinguish between OSI-420 and M13.

The in vitro model of HLM, resembling the liver in vivo conditions, was used to produce erlotinib metabolites. The essential cofactors, present in vivo, were added to the HLM for enzyme activation of the CYP and UGT enzymes. The effect of other metabolic enzymes cannot be studied using the model system used in this study.

In general, a good correlation between HLM generated metabolites and patient samples was observed but M6 was high in HLM whilst low in patient samples and vice versa for M3. M17 and M21 was equally distributed in HLM but M21 was the most abundant in patient samples.

The HLM generated samples identified all previously known erlotinib metabolites in healthy volunteers, except from a glucuronide M18 and a sulfate metabolite M9 [11]. Presumably M9 lacked the corresponding cofactor and M18 demanded longer incubation time or other environmental settings.

Three novel metabolites, M19, M20 and M21 were identified in HLM and were confirmed in patient samples. The structures were determined based on product ion spectra from LC-MS/MS.

Structure suggestions for M17 and its position isomer M21 was possible based on correlating mass spectra and relative retention times. The spectral differences between the position isomers, OSI420:M13 and M17:M21, might be caused by a more easily removed methoxyethane group compared to the ethanol group and that $\mathrm{R} 2$ is more exposed to fragmentation because of its three-dimensional orientation.

Long term stability of the glucuronides ( $M 3, M 8 / 10$ and $M 20$ ) could not be established in the validation process. M3 was exposed to degradation but no conversion into other metabolites was observed. The same degradation trend was observed for M8/10 and M20. Glucuronides are highly polar analytes in the later biotransformational steps before elimination and are in generally not active analytes and 
would therefore most likely not contribute to the clinical effect. To avoid glucuronide degradation, analysis shortly after sampling is recommended.

The metabolites M7, M16 and M11 are likely stable even though the CV is higher than expected. No degradation trends can be seen and the $\mathrm{CV}$ probably reflects method variation rather than stability issues.

The method was successfully applied to analyze clinical samples. Erlotinib was in the expected concentration range based on previously reports [12]. Mean concentrations of OSI-420 and M13 were $1.6 \%$ and $10.3 \%$ of erlotinib mean concentration respectively and combined they represent the expected percentage of erlotinib levels previously reported at steady state [12]. Didesmethyl erlotinib was present in low concentrations in human plasma, less than $1 \%$ of erlotinib, and have to the best of our knowledge not previously been reported. 


\section{Conclusions}

We developed a sensitive method for quantification of erlotinib, OSI-420 and didesmethyl erlotinib and semi-quantification of HLM generated metabolites. Of the 14 HLM generated metabolites, three novel metabolites were found and their structures determined. The method fulfilled all the requirements in international validation guidelines. With a simple sample preparation and a rapid seven minute analysis the method is suitable for clinical studies and TDM of erlotinib and its metabolites. 


\section{Acknowledgement}

We thank the personnel at the department of pulmonary medicine at the University Hospital in Linköping for assistance in collecting the clinical samples. We are also grateful to the Swedish Research Council, the Swedish Cancer Society and the Medical Research Council of Southeast Sweden for funding this study.

\section{Conflict of interest}

The authors have declared no competing interests. 


\section{References}

[1] J.D. Moyer, E.G. Barbacci, K.K. Iwata, L. Arnold, B. Boman, A. Cunningham, C. DiOrio, J. Doty, M.J. Morin, M.P. Moyer, M. Neveu, V.A. Pollack, L.R. Pustilnik, M.M. Reynolds, D. Sloan, A. Theleman, P. Miller, Induction of apoptosis and cell cycle arrest by CP-358,774, an inhibitor of epidermal growth factor receptor tyrosine kinase, CANCER RES., 57 (1997) 4838-4848.

[2] M. Hidalgo, L.L. Siu, J. Nemunaitis, J. Rizzo, L.A. Hammond, C. Takimoto, S.G. Eckhardt, A. Tolcher, C.D. Britten, L. Denis, K. Ferrante, D.D. Von Hoff, S. Silberman, E.K. Rowinsky, Phase I and pharmacologic study of OSI-774, an epidermal growth factor receptor tyrosine kinase inhibitor, in patients with advanced solid malignancies, J. Clin. Oncol., 19 (2001) 3267-3279.

[3] E. Petit-Jean, T. Buclin, M. Guidi, E. Quoix, B. Gourieux, L.A. Decosterd, A.C. Gairard-Dory, G. Ubeaud-Séquier, N. Widmer, Erlotinib: Another candidate for the therapeutic drug monitoring of targeted therapy of cancer? A pharmacokinetic and pharmacodynamic systematic review of literature, Ther. Drug Monit., (2014) http://dx.doi.org/10.1097/FTD.0000000000000097 [Epub ahead of print].

[4] C. Bardin, G. Veal, A. Paci, E. Chatelut, A. Astier, D. Levêque, N. Widmer, J. Beijnen, Therapeutic drug monitoring in cancer - Are we missing a trick?, European Journal of Cancer, 50 (2014) 2005-2009.

[5] G.M. Clark, D.M. Zborowski, P. Santabárbara, K. Ding, M. Whitehead, L. Seymour, F.A. Shepherd, Smoking History and Epidermal Growth Factor Receptor Expression as Predictors of Survival Benefit from Erlotinib for Patients with Non-Small-Cell Lung Cancer in the National Cancer Institute of Canada Clinical Trials Group Study BR.21, Clinical Lung Cancer, 7 (2006) 389-394.

[6] M. Hamilton, J.L. Wolf, J. Rusk, S.E. Beard, G.M. Clark, K. Witt, P.J. Cagnoni, Effects of smoking on the pharmacokinetics of erlotinib, Clin. Cancer Res., 12 (2006) 2166-2171.

[7] J. Ling, S. Fettner, B.L. Lum, M. Riek, A. Rakhit, Effect of food on the pharmacokinetics of erlotinib, an orally active epidermal growth factor receptor tyrosine-kinase inhibitor, in healthy individuals, Anti-Cancer Drugs, 19 (2008) 209-216.

[8] C.M. Rudin, W. Liu, A. Desai, T. Karrison, X. Jiang, L. Janisch, S. Das, J. Ramirez, B. Poonkuzhali, E. Schuetz, D.L. Fackenthal, P. Chen, D.K. Armstrong, J.R. Brahmer, G.F. Fleming, E.E. Vokes, M.A. Carducci, M.J. Ratain, Pharmacogenomic and pharmacokinetic determinants of erlotinib toxicity, J. Clin. Oncol., 26 (2008) 1119-1127.

[9] F. Thomas, P. Rochaix, M. White-Koning, I. Hennebelle, J. Sarini, A. Benlyazid, L. Malard, J.-L. Lefebvre, E. Chatelut, J.P. Delord, Population pharmacokinetics of erlotinib and its pharmacokinetic/pharmacodynamic relationships in head and neck squamous cell carcinoma, European Journal of Cancer, 45 (2009) 2316-2323.

[10] J. Li, M. Zhao, P. He, M. Hidalgo, S.D. Baker, Differential metabolism of gefitinib and erlotinib by human cytochrome P450 enzymes, Clin. Cancer Res., 13 (2007) 3731-3737.

[11] J. Ling, K.A. Johnson, Z. Miao, A. Rakhit, M.P. Pantze, M. Hamilton, B.L. Lum, C. Prakash, Metabolism and excretion of erlotinib, a small molecule inhibitor of epidermal growth factor receptor tyrosine kinase, in healthy male volunteers, Drug Metab. Dispos., 34 (2006) 420 426.

[12] W. Zhang, L.L. Siu, M.J. Moore, E.X. Chen, Simultaneous determination of OSI-774 and its major metabolite OSI-420 in human plasma by using HPLC with UV detection, Journal of Chromatography B, 814 (2005) 143-147.

[13] E.R. Lepper, S.M. Swain, A.R. Tan, W.D. Figg, A. Sparreboom, Liquid-chromatographic determination of erlotinib (OSI-774), an epidermal growth factor receptor tyrosine kinase inhibitor, J. Chromatogr. B Anal. Technol. Biomed. Life Sci., 796 (2003) $181-188$.

[14] L. Faivre, C. Gomo, O. Mir, F. Taieb, A. Schoemann-Thomas, S. Ropert, M. Vidal, D. Dusser, A. Dauphin, F. Goldwasser, B. Blanchet, A simple HPLC-UV method for the simultaneous quantification of gefitinib and erlotinib in human plasma, J. Chromatogr. B Anal. Technol. Biomed. Life Sci., 879 (2011) 2345-2350.

[15] S. Bouchet, E. Chauzit, D. Ducint, N. Castaing, M. Canal-Raffin, N. Moore, K. Titier, M. Molimard, Simultaneous determination of nine tyrosine kinase inhibitors by 96-well solid-phase extraction and ultra performance LC/MS-MS, Clin. Chim. Acta, 412 (2011) $1060-1067$.

[16] R. Honeywell, K. Yarzadah, E. Giovannetti, N. Losekoot, E.F. Smit, M. Walraven, J.S.W. Lind, C. Tibaldi, H.M. Verheul, G.J. Peters, Simple and selective method for the determination of various tyrosine kinase inhibitors used in the clinical setting by liquid chromatography tandem mass spectrometry, J. Chromatogr. B Anal. Technol. Biomed. Life Sci., 878 (2010) 1059-1068.

[17] A.R. Masters, C.J. Sweeney, D.R. Jones, The quantification of erlotinib (OSI-774) and OSI-420 in human plasma by liquid chromatography-tandem mass spectrometry, J. Chromatogr. B Anal. Technol. Biomed. Life Sci., 848 (2007) 379-383.

[18] M. Zhao, P. He, M.A. Rudek, M. Hidalgo, S.D. Baker, Specific method for determination of OSI-774 and its metabolite OSI-420 in human plasma by using liquid chromatography-tandem mass spectrometry, J. Chromatogr. B Anal. Technol. Biomed. Life Sci., 793 (2003) 413-420.

[19] L. Götze, A. Hegele, S.K. Metzelder, H. Renz, W.A. Nockher, Development and clinical application of a LC-MS/MS method for simultaneous determination of various tyrosine kinase inhibitors in human plasma, Clin. Chim. Acta, 413 (2012) 143-149.

[20] B. Mandal, P. Balabathula, N. Mittal, G.C. Wood, H. Bhattacharjee, Development and validation of a spectrofluorimetric method for the determination of erlotinib in spiked human plasma, J Fluoresc, 22 (2012) 1425-1429.

[21] J. Rodríguez, G. Castañeda, L. Muñoz, D. Navarro, J.C. Villa, Simultaneous determination of erlotinib and metabolites in human urine using capillary electrophoresis, Electrophoresis, 35 (2014) 1489-1495. 
[22] U.S Department of Health and Human Services, Food and Drug Administration, Center for Drug Evaluation and Research (CDER), Center for Veterinary Medicine (CVM), Guidance for Industry Bioanalytical Method Validation, (2001).

[23] European Medicines Agency (EMA), Committee for Medicinal Products or Human Use (CHMP), Guideline on Bioanalytical Method Validation, (2011).

[24] L. Signor, E. Varesio, R.F. Staack, V. Starke, W.F. Richter, G. Hopfgartner, Analysis of erlotinib and its metabolites in rat tissue sections by MALDI quadrupole time-of-flight mass spectrometry, J. Mass Spectrom., 42 (2007) 900-909. 